\title{
The Role of Glucocorticoids in the Management of COVID-19
}

\section{(ㄷ) (i) 응 $\ominus$}

\author{
Authors \\ Vasileia Ismini Alexaki ${ }^{1}$, Holger Henneicke2, 3
}

\begin{abstract}
Affiliations
1 Institute for Clinical Chemistry and Laboratory Medicine, Faculty of Medicine and University Clinic Carl Gustav Carus, TU Dresden, Dresden, Germany

2 Center for Regenerative Therapies Dresden, TU Dresden, Dresden, Germany

3 Department of Medicine III \& Center for Healthy Aging, University Clinic Carl Gustav Carus, TU Dresden, Dresden, Germany
\end{abstract}

Key words

glucocorticoids inflammation COVID-19

received 21.09.2020

accepted after revision $\quad 14.10 .2020$

published online 19.11 .2020

Bibliography

Horm Metab Res 2021; 53: 9-15

DOI $10.1055 / \mathrm{a}-1300-2550$

ISSN 0018-5043

(C) 2020. The Author(s).

This is an open access article published by Thieme under the terms of the Creative Commons Attribution-NonDerivative-NonCommercial-License, permitting copying and reproduction so long as the original work is given appropriate credit. Contents may not be used for commecial purposes, or adapted, remixed, transformed or built upon. (https://creativecommons.org/ licenses/by-nc-nd/4.0/)

Georg Thieme Verlag KG, Rüdigerstraße 14,

70469 Stuttgart, Germany

Correspondence

Dr. V. I. Alexaki

Institute for Clinical Chemistry and Laboratory Medicine,

University Clinic Carl Gustav Carus, TU Dresden

Fetscherstrasse 74

01307 Dresden

Germany

Tel.: +49351458 16273, Fax: +4935145816324

Vasileialsmini.Alexaki@uniklinikum-dresden.de
Dr. H. Henneicke

Center for Regenerative Therapies Dresden, TU Dresden

Fetscherstrasse 74

01307 Dresden

Germany

Tel.: + 4935145819536 ,

holger.henneicke@uniklinikum-dresden.de

\section{ABSTRACT}

Coronavirus disease 2019 (COVID-19), caused by an infection with the novel coronavirus SARS-CoV-2, has resulted in a global pandemic and poses an emergency to public health systems worldwide. COVID-19 is highly infectious and is characterized by an acute respiratory illness that varies from mild flu-like symptoms to the life-threatening acute respiratory distress syndrome (ARDS). As such, there is an urgent need for the development of new therapeutic strategies, which combat the high mortality in severely ill COVID-19 patients. Glucocorticoids are a frontline treatment for a diverse range of inflammatory diseases. Due to their immunosuppressive functions, the use of glucocorticoids in the treatment of COVID-19 patients was initially regarded with caution. However, recent studies concluded that the initiation of systemic glucocorticoids in patients suffering from severe and critical COVID-19 is associated with lower mortality. Herein we review the anti-inflammatory effects of glucocorticoids and discuss emerging issues in their clinical use in the context of COVID-19.

\section{Introduction}

Coronavirus disease 2019 (COVID-19), first reported at the end of 2019 in Wuhan, China, rapidly spread around the globe and was declared a pandemic by the WHO in March 2020 [1]. As of October
2020, more than 30000000 cases and 950000 deaths were reported worldwide. COVID-19 is caused by the severe acute respiratory syndrome coronavirus 2 (SARS-CoV-2). SARS-CoV-2 shows close relation to SARS-CoV, the underlying viral cause of the 2002 
SARS epidemic, as well as to MERS-CoV, which causes sporadic outbreaks centered in the Middle East [2]. COVID-19 patients develop an acute respiratory illness, which may cause mild fevers and coughs but may also lead to acute respiratory distress syndrome (ARDS), hypoxic respiratory failure, and ultimately death [3]. Immunologically, critical cases of COVID-19 are signified by an excessive inflammatory response, manifested by strong leukocyte influx into the lungs and increased plasma levels of inflammatory markers [4]. Additionally, coagulopathies and increased risk of blood clot formation have been reported as significant contributors of COVID-19 related mortality [5]. The excessive inflammatory response observed in severe and critical COVID-19 patients raised the possibility of the use of anti-inflammatory drugs to combat the excessive and damaging immune response. While medical professionals were apprehensive about the use of immunosuppressants to treat an infectious disease in the early stages of the pandemic, over time, evidence accrued that glucocorticoids lead to improved patient outcomes [6].

\section{Glucocorticoids and the glucocorticoid receptor (GR)}

Glucocorticoids are stress hormones synthesized in the adrenal cortex under the control of the hypothalamic-pituitary-adrenal (HPA) axis [7, 8]. Under normal conditions glucocorticoids are released in a circadian and ultradian rhythm $[7,8]$. Stress stimuli activate the HPA axis: in particular pro-inflammatory cytokines, such as IL-6, TNF, and IL-1 $\beta$, act on the hypothalamus triggering HPA axis activation and leading to adrenal glucocorticoid production [7-9]. In turn, circulating glucocorticoids exert strong immunomodulatory effects and constrain inflammation, while also limiting their own production through a negative feedback on the HPA axis $[7,8,10]$. Following their release into the bloodstream glucocorticoid tissue availability and function are determined by several regulatory mechanisms. Corticosteroid-binding globulin (CBG) binds and keeps approximately $90 \%$ of cortisol in an inactive state. Neutrophil elastase cleaves CBG, thereby releasing bioactive glucocorticoids at sites of inflammation $[7,8]$. Moreover, $11 \beta$-hydroxysteroid dehydrogenases 1 and $2(11 \beta-H S D 1,11 \beta$-HSD2) regulate the interconversion of bioactive cortisol to inactive cortisone in target tissues [7]. Inflammatory signals can regulate the expression of $11 \beta$ HSD1 and 2, which determines local glucocorticoid activity [8].

Glucocorticoids are lipophilic molecules diffusing through the cell membrane and binding to the cytosolic glucocorticoid receptor (GR), which is abundantly expressed in most cell types [7]. The GR contains an $\mathrm{N}$-terminal domain, which interacts with co-factors and transcription factors, a zinc-finger motif-containing DNA-binding domain and a ligand-binding domain $[7,11]$. The GR is subjected to post-translational modifications, including phosphorylation, acetylation, ubiquitylation, and sumoylation at multiple sites, which regulate its compartmentalization, function and degradation [11]. While GR $\alpha$ is the ligand-binding GR isoform, its splice isoform GR $\beta$ does not bind glucocorticoids and exerts dominant-negative effects on GR $\alpha$. In its inactive state GR forms complexes with heat-shock proteins, chaperones, and immunophilins. Upon ligation the receptor undergoes conformational changes, partially disassembles from its interactors, translocates to the nucleus, and controls gene transcription as a monomer or dimer [7]. Upon its translocation to the nucleus, GR can directly bind to glucocorticoid response elements (GRE) to induce or inhibit gene expression [7]. High glucocorticoid doses favor binding of GR homodimers rather than monomers on palindromic GRE [12]. In addition, GR can tether transcription factors through protein-protein interactions without the requirement of DNA binding [13]. Through the latter mechanism GR can block the function of transcription factors mediating inflammatory responses, such as nuclear factor-kB (NF-kB) and activator protein 1 (AP-1) [8]. However, transrepression can also be mediated by competition for binding to the DNA or for co-factors [7]. Moreover, GR directly interacts with NF-кB, hindering its translocation to the nucleus and induces the expression of the NF- $\mathrm{kB}$ inhibitor glucocorticoid-induced leucine zipper protein (GILZ) $[14,15]$.

Additionally to the GR-mediated genomic effects, glucocorticoids may also exert non-genomic effects, leading to rapid biological events $[7,16]$. This mechanism of action has been shown to contribute to the effects of glucocorticoids on T-cell receptor (TCR) signaling, neutrophil degranulation, macrophage inflammatory activation, mast cell-mediated histamine release and ion channel function in bronchial epithelial cells [16-18].

\section{Anti-inflammatory effects of glucocorticoids}

Glucocorticoids regulate inflammation through pleiotropic mechanisms $[8,19]$. Their effects on immune responses depend on the cell type, disease, dose and timing of application, that is, whether application precedes or follows exposure to the inflammatory stimulus [20-22]. While exposure to low doses of glucocorticoids prior to a noxious stimulus can promote the inflammatory response, glucocorticoids in high doses applied after the inflammatory stimulus act in an anti-inflammatory fashion [8,19-22]. In the absence of inflammation, glucocorticoids promote the expression of pattern recognition receptors (PRR), such as Toll-like receptor (TLR), thereby maintaining the cells sensitivity to noxious stimuli, such as pathogen- and damage-associated molecular patterns (PAMP, DAMP) $[8,19]$. In the context of an acute inflammatory response glucocorticoids taper inflammation through several mechanisms. Glucocorticoids inhibit PRR signaling by tethering NF- $\mathrm{KB}$ and AP-1 and induce the expression of negative regulators of TLR signaling, such as dual-specificity protein phosphatase 1 (DUSP1) and IL-1 Receptor-Associated Kinase M (IRAK-M) [8, 23]. In macrophages glucocorticoids down-regulate the expression of pro-inflammatory cytokines, such as IL-1 $\beta$, IL-6, IL-12, IL-17, TNF, and granulocyte-macrophage colony-stimulating factor (GMCSF), and inducible nitric oxide synthase (iNOS) and inhibit cyclooxygenase 2 in a GILZ-dependent manner, thereby attenuating release of prostaglandins [8, 22, 24-27]. Moreover, in endothelial cells they inhibit the expression of adhesion molecules, like E-selectin, intercellular adhesion molecule 1 (ICAM1) and vascular cell adhesion molecule 1 (VCAM1). In immune cells they down-regulate the expression of integrins, such as lymphocyte function-associated antigen 1 (LFA1) and very late antigen 4 (VLA4), thereby attenuating leukocyte recruitment $[8,28,29]$. Glucocorticoids also shift macrophages towards an anti-inflammatory state, which secretes more IL-10 and transforming growth factor- $\beta$ (TGF $\beta$ ), and promote efferocytosis and cell debris clearance due to increased expression of scavenger molecules (CD163, CD206 and tyrosine-protein kinase MER (MERTK)), thereby favoring resolution of inflammation $[8,19,22,30-35]$. The 
reaction to antigens is regulated by glucocorticoids at both, the level of antigen presentation and $T$ cell activation. Glucocorticoids inhibit maturation, promote apoptosis and attenuate antigen presentation in dendritic cells $[8,36,37]$. Glucocorticoids also regulate thymopoiesis through induction of lymphocyte apoptosis and attenuate T cell receptor signaling [38, 39]. Moreover, glucocorticoids increase regulatory $T$ cell (Tregs) frequency through GILZ and TGFß-dependent induction of Forkhead box P3 (FoxP3), a transcription factor determining Treg differentiation [40].

Overall, therapeutic glucocorticoids exert potent anti-inflammatory effects across both the innate and adaptive immune system. Due to these wide-ranging anti-inflammatory effects glucocorticoids are a mainstay of immunosuppressive therapies. Synthetic glucocorticoid compounds, such as dexamethasone and prednisolone, show increased potency compared to cortisol owing to longer half-life in plasma, improved parenteral absorption and reduced binding to CBG. However, due to their wide-ranging receptor expression and the large-scale GR-mediated transcriptional changes - overall approximately $20 \%$ of the genome is responsive to $G R$ - glucocorticoids are not only potent immunosuppressants, but also exert broad off-target effects [7,41,42].

\section{Adverse effects of glucocorticoids}

Adverse effects related to glucocorticoid treatment are common and numerous, and depend on the dose and duration of therapy $[7,42,43]$. A number of glucocorticoid side effects are a result of glucocorticoid-mediated inhibition of glucose uptake and other metabolic alterations of basic cellular metabolism, which typically occur in most cells and tissues [42,43]. Common side effects include weight gain and the development of diabetes mellitus, sarcopenia, and osteoporosis. Also, an increase in the incidence of hypertension, atherosclerosis, cardiovascular disease, and thromboembolism may be observed. Additionally, therapeutic doses of glucocorticoids raise susceptibility to infections and can cause impaired wound healing, psychiatric disturbances, and suppression of the HPA axis with the risk of secondary adrenal insufficiency $[42,44]$.

\section{COVID-19 immunopathogenesis and glucocorticoids}

Following priming of the viral spike $(S)$ protein through the serine protease TMPRSS2 [45], SARS-CoV-2 gains access to respiratory epithelial cells through the hosts angiotensin-converting enzyme 2 (ACE2) protein [4]. Upon cellular entry, the viral RNA genome is released into the host cell and new virions are produced [46]. The viral capture of the host cell is followed by the initiation of an innate immune response, which features inflammatory cytokine and chemokine production, coupled to neutrophil and monocyte infiltration of the respiratory tract and lungs. This inflammatory response is typically associated with increased capillary leakage and alveolar cell destruction, damaging respiratory function. Systemic inflammation is reflected by increased plasma concentrations of inflammatory cytokines, such as IL-1 $\beta$, IL-6, IL-8, IL-10, IL-17, IP10, and TNF, in COVID-19 patients, whereas levels of type I and III interferons remain low [47-49]. Increased circulating neutrophil numbers correlate with poor prognosis in COVID-19 and strong neutrophil infiltration of pulmonary capillaries was reported in postmortem lung tissues $[47,50]$. The complement system is ac- tivated during ARDS and activation of $C 3$ exacerbates disease in SARS-CoV-associated ARDS [51,52]. Thus, the complement system may drive part of the inflammatory response and thrombosis to COVID-19 [51, 53-55]. Observational studies suggest that, elevated levels of $C$-reactive protein (CRP) and lactate dehydrogenase are predictors of COVID-19 severity [4, 47]; similar correlations have been established for elevated neutrophil/lymphocyte ratio, low platelet count and increased numbers of $\mathrm{CD} 14^{+} \mathrm{CD} 16^{+}$monocytes and Th 17 cells $[4,56,57]$.

Due to the rapidly developing nature of the COVID-19 pandemic studies examining the molecular effects of glucocorticoids in patients suffering from severe disease are few and far between. Based on the known and extensive immunosuppressive effects of glucocorticoids outlined above, corticosteroid treatment likely attenuates the COVID-19 induced inflammatory response - particularly in severely affected patients. Indeed, administration of glucocorticoids was reported to reduce CRP and IL-6 plasma levels in COVID19 patients, without affecting virus clearance $[58,59]$. Future studies identifying glucocorticoid target cells and anti-inflammatory mechanisms in the context of COVID-19 are critical in order enable the development of targeted therapies in the future.

\section{The clinical use of glucocorticoids in acute respiratory distress syndrome (ARDS) and COVID-19}

ARDS is primarily signified by hypoxic respiratory failure. In general, ARDS is associated with increased vascular permeability and pulmonary edema as well as increased systemic inflammation, frequently leading to multi-organ failure and death. Thus, ARDS-related mortality is as high as $50 \%$ [60].

Treatment of ARDS is primarily focused on a combination of low tidal volume mechanical ventilation and prone positioning [61, 62]. Even though inflammation likely plays a key role in the pathogenesis of ARDS, anti-inflammatory therapeutic strategies using glucocorticoids have yielded mixed results. For example, two meta-analysis examining the subject of glucocorticoid use in ARDS a decade ago reached opposite conclusions ranging from advising against their use to recommending steroid treatment in ARDS $[63,64]$. These inconsistent conclusions arose from original research studies, which included small numbers of patients, and varied in their definition of ARDS, type of steroid used, as well as timing and dosing strategies. In order to address this issue efforts have been made to clarify the effectiveness of glucocorticoids in ARDS through large-scale multi-centered randomized controlled trials. The recent Dexa-ARDS trial found that patients receiving high-dose dexamethasone had a lower all-cause mortality than patients receiving standard care (21\% vs. $36 \%$ respectively) [64]. A meta-analysis, which included the Dexa-ARDS trial, as well as others, also favored the use of glucocorticoids in ARDS, although some reservations remained regarding the reliability of the findings [65]. Additionally, a recent meta-analysis attempted to dissect the issues of timing and dosing of glucocorticoid use in ARDS and came to the conclusion that early initiation of glucocorticoid therapy and use of a low to medium dose were associated with lower mortality [66]. Overall, the issue of glucocorticoid use in ARDS is still not entirely resolved, however, recent evidence has tipped the balance in favor of glucocorticoid treatment with a recommendation for early commencement of steroids at a low to medium dose. Of note, the 
large-scale LUNG SAFE study of 2377 patients with ARDS found an overall rate of glucocorticoid use of $<20 \%$ [60].

With the beginning of the COVID-19 pandemic, medical professionals were at a loss as to whether glucocorticoids could have a role in treating severely affected patients, particularly in those suffering from hypoxic respiratory failure and ARDS. In the absence of studies examining the effects of glucocorticoids in COVID-19 patients specifically, doctors were apprehensive to utilize a potent immunosuppressive agent to treat an infectious disease. Evidence originating from the SARS und MERS outbreaks showed a delayed virus clearance in patients receiving glucocorticoids potentially indicating an impaired host response to the viral infection [67]. Moreover, the use of steroids in the treatment of influenza has been studied to some degree, and while the evidence is far from conclusive, the general consensus does not recommend the use of glucocorticoids in patients suffering from influenza infections [68]. Adding to the concern was an increased risk of bacterial superinfection, which typically is associated with the use of medium to high dose glucocorticoids [69, 70].

Following encouraging case reports and small observational studies using glucocorticoids in severely affected COVID-19 patients, multiple large-scale RCTs were initiated, most notably the RECOVERY trial in the United Kingdom [71]. As part of this study 2104 intrahospital COVID-19 patients received $6 \mathrm{mg}$ of dexamethasone for 10 days (or until discharge from hospital, if sooner), while 4321 patients received standard care [72]. Overall the mortality rate after 28 days was significantly lower in the Dexamethasone group (22.9 vs. $25.7 \%$ ). Interestingly, subgroup analysis revealed that patients requiring mechanical ventilation benefited the most from the pharmacological intervention with glucocorticoids. In this group of patients, the mortality after 28 days was reduced by more than 12.1 percentage points in the dexamethasone group ( $29.3 \mathrm{vs.}$ $41.4 \%)$. Dexamethasone also conferred a survival benefit in patients requiring oxygen - without the need for invasive mechanical ventilation - however, the effect remained small (28-day mortality: $23.3 \%$ vs. $26.2 \%)$. In patients who did not require ventilation support or oxygen Dexamethasone showed no significant effect. Following the publication of the RECOVERY data as a preliminary report, treatment guidelines were updated to recommend the use of glucocorticoids in severely affected COVID-19 patients. This also led to an arrest of patient recruitment for other ongoing trials examining the effects of glucocorticoids for the treatment of (severe) COVID-19.

The Rapid Evidence Appraisal for COVID-19 Therapies (REACT) Working Group of the WHO performed a meta-analysis of all available data regarding the use of glucocorticoids in COVID-19 [6]. The analysis included the RECOVERY trial as well as 6 other trials - some of which had to be abrogated before reaching their recruitment goal due to the publication of the RECOVERY data. The meta-analysis concluded that initiation of systemic glucocorticoids was associated with lower 28-day all-cause mortality in critically ill patients with COVID-19. Consequently, based on these and other studies, the $\mathrm{WHO}$ issued two recommendations regarding the treatment of COVID-19: First, systemic glucocorticoid therapy should be initiated in patients with severe and critical COVID-19, whereby therapy is defined as $6 \mathrm{mg}$ daily of dexamethasone orally or intravenously or $50 \mathrm{mg}$ of hydrocortisone intravenously every 8 hours for

- Table 1 Mutually exclusive categories of illness severity.

Critical COVID-19

Severe COVID-19

Non-severe COVID-19

Reference: Corticosteroids for COVID-19, WHO, 2. September 2020.

7 to 10 days. Secondly, the use of glucocorticoids in non-severe COVID-19 is not advised (for definition of critical, severe and non-critical COVID-19, see $>$ Table 1).

\section{Glucocorticoids, COVID-19 and metabolic comorbidities}

Diabetes mellitus is associated with an increased risk of infection and infection-related death $[73,74]$. Accordingly, diabetic patients were at an increased risk of severe or lethal infection for both SARS as well as MERS during the respective outbreaks [75, 76]. When the Sars-Cov-2 spread around the world it quickly emerged that in the country of origin, China, diabetes was associated with increased mortality due to COVID-19 [77] - a finding later confirmed elsewhere [78]. Amongst patients suffering from diabetes mellitus, COVID-19 related mortality was not only associated with the presence of cardiovascular complications but also with glycemic control and BMI $[79,80]$. An elevated BMI as well as obesity are also associated with severe outcomes of COVID-19 such ICU admission and mechanical ventilation in non-diabetic patients, thus indicating their role as an independent risk factors for COVID-19 related mortality [81, 82]. The association between good glycemic control and survival of COVID-19 amongst diabetics highlights the importance of adequate control of blood sugar levels during Sars-CoV-2 infection. As a modifiable risk factor, this association has already led to expert recommendations for the management of diabetes in patients with COVID-19 [83]. The reasons for increased mortality from COVID-19 in diabetic patients and the potential contribution of blood sugar levels to infection outcomes remain largely obscure. Multiple potential molecular mechanisms have been described and include a diabetes associated (i) increase in Coronavirus 
load, (ii) dysregulated immune response, (iii) alveolar dysfunction, (iv) epithelial dysfunction and (v) coagulopathy [84]. However, the relative importance of these molecular phenomena needs to be investigated further and clinically validated. Interestingly, to our current knowledge, no study investigated the efficiency of glucocorticoid treatment in diabetic COVID-19 patients specifically. While the overall benefit of glucocorticoid therapy in severe and critical COVID-19 infections has been established, the efficiency of glucocorticoids in diabetics is currently less clear. Thus far - to the best of our knowledge - no subgroup analysis has examined the effect of glucocorticoids in diabetic patients suffering from COVID-19. This question is of particular relevance as exposure to supraphysiological levels of glucocorticoids frequently leads to worse glycemic control $[85,86]$. Additionally, it is well established that medium to high doses of therapeutic glucocorticoids cause hypertension $[87,88]$, weight gain [89] as well as an increase in cardiovascular events $[90,91]$, all of which are associated with worse outcomes in COVID-19. Whether the adverse effects of therapeutic glucocorticoids are of concern during short exposure of 7-10 days (as recommended for COVID-19 treatment) remains to be evaluated.

\section{Conclusion}

Recent large-scale clinical trials support the use of glucocorticoids in the treatment of both ARDS as well as severe and critical COVID-19 infections. The use of glucocorticoids in high-risk COVID-19 patients suffering from diabetes mellitus and additional co-morbidities such as obesity, hypertension, and cardiovascular disease should be evaluated in further clinical trials. Furthermore, COVID-19 patients treated with glucocorticoids should undergo close immunological characterization in order to enable identification of molecular pathways for the development of targeted therapies.

\section{Funding Information}

This work was supported by funds from the Deutsche Forschungsgemeinschaft (SFB-TRR 205 to V.I.A. \& HE 8391/1-1 to H.H.).

\section{Conflict of Interest}

V.I.A. declares no conflict of interest. H.H. has received speaking fees from Amgen and has served on scientific advisory boards for Novo Nordisk and Takeda.

\section{References}

[1] Chen N, Zhou M, Dong X et al. Epidemiological and clinical characteristics of 99 cases of 2019 novel coronavirus pneumonia in Wuhan, China: A descriptive study. Lancet 2020; 395: 507-513

[2] Lu R, Zhao X, Li J et al. Genomic characterisation and epidemiology of 2019 novel coronavirus: implications for virus origins and receptor binding. Lancet 2020; 395: 565-574

[3] Fan E, Beitler JR, Brochard L et al. COVID-19-associated acute respiratory distress syndrome: Is a different approach to management warranted? Lancet Respir Med 2020; 8: 816-821
[4] Hussman JP. Cellular and Molecular Pathways of COVID-19 and potential points of therapeutic intervention. Front Pharmacol 2020; 11: 1169

[5] Shah A, Donovan K, McHugh A et al. Thrombotic and haemorrhagic complications in critically ill patients with COVID-19: A multicentre observational study. Crit Care 2020; 24: 561

[6] Group WHOREAfC-TW Sterne JAC, Murthy S et al. Association between administration of systemic corticosteroids and mortality among critically ill patients with COVID-19: A Meta-analysis. JAMA 2020; 324: 1330-1341

[7] Timmermans S, Souffriau ], Libert C. A General Introduction to Glucocorticoid Biology. Front Immunol 2019; 10: 1545

[8] Cain DW, Cidlowski JA. Immune regulation by glucocorticoids. Nat Rev Immunol 2017; 17: 233-247

[9] Kanczkowski W, Alexaki VI, Tran N et al. Hypothalamo-pituitary and immune-dependent adrenal regulation during systemic inflammation. Proc Natl Acad Sci USA 2013; 110: 14801-14806

[10] Vandewalle J, Libert C. Glucocorticoids in Sepsis: To Be or Not to Be. Front Immunol 2020; 11: 1318

[11] Petta I, Dejager L, Ballegeer M et al. The interactome of the glucocorticoid receptor and its influence on the actions of glucocorticoids in combatting inflammatory and infectious diseases. Microbiol Mol Biol Rev 2016; 80: 495-522

[12] Lim HW, Uhlenhaut NH, Rauch A et al. Genomic redistribution of GR monomers and dimers mediates transcriptional response to exogenous glucocorticoid in vivo. Genome Res 2015; 25: 836-844

[13] Hudson WH, Youn C, Ortlund EA. The structural basis of direct glucocorticoid-mediated transrepression. Nat Struct Mol Biol 2013; 20: $53-58$

[14] Widen C, Gustafsson JA, Wikstrom AC. Cytosolic glucocorticoid receptor interaction with nuclear factor-kappa $B$ proteins in rat liver cells. Biochem J 2003; 373: 211-220

[15] Berrebi D, Bruscoli S, Cohen N. Synthesis of glucocorticoid-induced leucine zipper (GILZ) by macrophages: an anti-inflammatory and immunosuppressive mechanism shared by glucocorticoids and IL-10. Blood 2003; 101: 729-738

[16] Panettieri RA, Schaafsma D, Amrani Y et al. Non-genomic effects of glucocorticoids: An updated view. Trends Pharmacol Sci 2019; 40: 38-49

[17] Lowenberg M, Verhaar AP, Bilderbeek J et al. Glucocorticoids cause rapid dissociation of a T-cell-receptor-associated protein complex containing LCK and FYN 2006; 7: 1023-1029

[18] Jiang CL, Liu L, Tasker JG. Why do we need nongenomic glucocorticoid mechanisms? Front Neuroendocrinol 2014; 35: 72-75

[19] Busillo JM, Cidlowski JA. The five Rs of glucocorticoid action during inflammation: Ready, reinforce, repress, resolve, and restore. Trends Endocrinol Metab 2013; 24: 109-119

[20] Lim HY, Muller N, Herold M] et al. Glucocorticoids exert opposing effects on macrophage function dependent on their concentration. Immunology 2007; 122: 47-53

[21] Dhabhar FS, McEwen BS. Enhancing versus suppressive effects of stress hormones on skin immune function. Proc Natl Acad Sci USA 1999; 96 : 1059-1064

[22] Quatrini L, Ugolini S. New insights into the cell- and tissue-specificity of glucocorticoid actions. Cell Mol Immunol 2020; 1-10

[23] Miyata M, Lee JY, Susuki-Miyata S et al. Glucocorticoids suppress inflammation via the upregulation of negative regulator IRAK-M. Nat Commun 2015; 6: 6062

[24] Bhattacharyya S, Brown DE, Brewer JA et al. Macrophage glucocorticoid receptors regulate Toll-like receptor 4-mediated inflammatory responses by selective inhibition of p38 MAP kinase. Blood 2007; 109: 4313-4319 
[25] Visser ], van Boxel-Dezaire A, Methorst D et al. Differential regulation of interleukin-10 (IL-10) and IL-12 by glucocorticoids in vitro. Blood 1998; 91: 4255-4264

[26] Lee SH, Soyoola E, Chanmugam P et al. Selective expression of mitogen-inducible cyclooxygenase in macrophages stimulated with lipopolysaccharide. J Biol Chem 1992; 267: 25934-25938

[27] Yang N, Zhang W, Shi XM. Glucocorticoid-induced leucine zipper (GILZ) mediates glucocorticoid action and inhibits inflammatory cytokine-induced COX-2 expression. J Cell Biochem 2008; 103: 1760-1771

[28] Mitroulis I, Alexaki VI, Kourtzelis I et al. Leukocyte integrins: role in leukocyte recruitment and as therapeutic targets in inflammatory disease. Pharmacol Ther 2015; 147: 123-135

[29] Zielinska KA, Van Moortel L, Opdenakker G et al. Endothelial response to glucocorticoids in inflammatory diseases. Front Immunol 2016; 7: 592

[30] Ehrchen J, Steinmuller L, Barczyk K et al. Glucocorticoids induce differentiation of a specifically activated, anti-inflammatory subtype of human monocytes. Blood 2007; 109: 1265-1274

[31] Mozo L, Suarez A, Gutierrez C. Glucocorticoids up-regulate constitutive interleukin-10 production by human monocytes. Clin Exp Allergy 2004; 34: 406-412

[32] Tu GW, Shi Y, Zheng Y] et al. Glucocorticoid attenuates acute lung injury through induction of type 2 macrophage. J Transl Med 2017; 15 : 181

[33] Meers GK, Bohnenberger H, Reichardt HM et al. Impaired resolution of DSS-induced colitis in mice lacking the glucocorticoid receptor in myeloid cells. PLoS One 2018; 13: e0190846

[34] Vallelian F, Schaer CA, Kaempfer T et al. Glucocorticoid treatment skews human monocyte differentiation into a hemoglobin-clearance phenotype with enhanced heme-iron recycling and antioxidant capacity. Blood 2010; 116: 5347-5356

[35] van der Goes A, Hoekstra K, van den Berg TK et al. Dexamethasone promotes phagocytosis and bacterial killing by human monocytes/ macrophages in vitro. J Leukoc Biol 2000; 67: 801-807

[36] Cao Y, Bender IK, Konstantinidis AK et al. Glucocorticoid receptor translational isoforms underlie maturational stage-specific glucocorticoid sensitivities of dendritic cells in mice and humans. Blood 2013; 121: 1553-1562

[37] Kim KD, Choe YK, Choe IS et al. Inhibition of glucocorticoid-mediated, caspase-independent dendritic cell death by CD40 activation. J Leukoc Biol 2001; 69: 426-434

[38] Wang D, Muller N, McPherson KG et al. Glucocorticoids engage different signal transduction pathways to induce apoptosis in thymocytes and mature T cells. J Immunol 2006; 176: 1695-1702

[39] Lowenberg M, Verhaar AP, van den Brink GR et al. Glucocorticoid signaling: a nongenomic mechanism for T-cell immunosuppression. Trends Mol Med 2007; 13: 158-163

[40] Bereshchenko O, Coppo M, Bruscoli S et al. GILZ promotes production of peripherally induced Treg cells and mediates the crosstalk between glucocorticoids and TGF-beta signaling. Cell Rep 2014; 7: 464-475

[41] Galon J, Franchimont D, Hiroi N et al. Gene profiling reveals unknown enhancing and suppressive actions of glucocorticoids on immune cells. FASEB J 2002; 16: 61-71

[42] Alan I, Alan B. Side effects of glucocorticoids. Pharmacokinetics and adverse effects of drugs - mechanisms and risks Factors. 2017; DOI: 10.5772/intechopen.72019

[43] Huscher D, Thiele K, Gromnica-Ihle E et al. Dose-related patterns of glucocorticoid-induced side effects. Ann Rheum Dis 2009; 68: 1119-1124

[44] Nicolaides NC, Pavlaki AN, Maria Alexandra MA et al.Eds Endotext. South Dartmouth, MA: 2000
[45] Hoffmann M, Kleine-Weber H, Schroeder S et al. SARS-CoV-2 cell entry depends on ACE2 and TMPRSS2 and Is blocked by a clinically proven protease inhibitor. Cell 2020; 181: 271-280 e278

[46] Shereen MA, Khan S, Kazmi A. COVID-19 infection: Origin, transmission, and characteristics of human coronaviruses. J Adv Res 2020; 24: 91-98

[47] Huang C, Wang Y, Li X et al. Clinical features of patients infected with 2019 novel coronavirus in Wuhan, China. Lancet 2020; 395: 497-506

[48] Qin C, Zhou L, Hu Z et al. Dysregulation of Immune Response in Patients With Coronavirus 2019 (COVID-19) in Wuhan, China. Clin Infect Dis 2020; 71: 762-768

[49] Blanco-Melo D, Nilsson-Payant BE, Liu WC et al. Imbalanced Host Response to SARS-CoV-2 Drives Development of COVID-19. Cell 2020; 181: 1036-1045 e1039

[50] Fox SE, Akmatbekov A, Harbert JL et al. Pulmonary and cardiac pathology in African American patients with COVID-19: An autopsy series from New Orleans. Lancet. Respir Med 2020; 8: 681-686

[51] Polycarpou A, Howard M, Farrar CA et al. Rationale for targeting complement in COVID-19. EMBO Mol Med 2020; 12: e12642

[52] Gralinski LE, Sheahan TP, Morrison TE et al. Complement Activation Contributes to Severe Acute Respiratory Syndrome Coronavirus Pathogenesis. mBio 2018; 9: e01753-18

[53] Risitano AM, Mastellos DC, Huber-Lang M et al. Complement as a target in COVID-19? Nat Rev Immunol 2020; 20: 343-344

[54] Java A, Apicelli AJ, Liszewski MK et al. The complement system in COVID-19: friend and foe? JCl Insight 2020; 5: e140711

[55] Risitano AM, Mastellos DC, Huber-Lang M et al. Author Correction: Complement as a target in COVID-19? Nat Rev Immunol 2020; 20: 448

[56] Xu Z, Shi L, Wang Y et al. Pathological findings of COVID-19 associated with acute respiratory distress syndrome. Lancet. Respir Med 2020; 8: 420-422

[57] Lippi G, Plebani M, Henry BM. Thrombocytopenia is associated with severe coronavirus disease 2019 (COVID-19) infections: A meta-analysis. Clin Chim Acta 2020; 506: 145-148

[58] Liu F, Ji C, Luo J et al. Clinical characteristics and corticosteroids application of different clinical types in patients with corona virus disease 2019. Sci Rep 2020; 10: 13689

[59] Selvaraj V, Dapaah-Afriyie K, Finn A et al. Short-Term Dexamethasone in Sars-CoV-2 Patients. R I Med J (2013) 2020; 103: 39-43

[60] Bellani G, Laffey JG, Pham T. Epidemiology, patterns of care, and mortality for patients with acute respiratory distress syndrome in intensive care units in 50 countries. JAMA 2016; 315: 788-800

[61] Acute Respiratory Distress Syndrome N, Brower RG, Matthay MA et al. Ventilation with lower tidal volumes as compared with traditional tidal volumes for acute lung injury and the acute respiratory distress syndrome. N Engl J Med 2000; 342: 1301-1308

[62] Guerin C, Reignier J, Richard JC. Prone positioning in severe acute respiratory distress syndrome. N Engl J Med 2013; 368: 2159-2168

[63] Agarwal R, Nath A, Aggarwal AN et al. Do glucocorticoids decrease mortality in acute respiratory distress syndrome? A meta-analysis. Respirology 2007; 12: 585-590

[64] Meduri GU, Marik PE, Chrousos GP et al. Steroid treatment in ARDS: a critical appraisal of the ARDS network trial and the recent literature. Intensive Care Med 2008; 34: 61-69

[65] Zayed Y, Barbarawi M, Ismail E et al. Use of glucocorticoids in patients with acute respiratory distress syndrome: A meta-analysis and trial sequential analysis. J Intensive Care 2020; 8: 43

[66] Zhao Q, Shi JX, Hu R et al. Effect of glucocorticoids on mortality in patients with acute respiratory distress syndrome: A meta-analysis. Exp Ther Med 2019; 18: 4913-4920 
[67] Arabi YM, Mandourah Y, Al-Hameed F et al. Corticosteroid Therapy for Critically III Patients with Middle East Respiratory Syndrome. Am J Respir Crit Care Med 2018; 197: 757-767

[68] Lansbury LE, Rodrigo C, Leonardi-Bee J et al. Corticosteroids as adjunctive therapy in the treatment of influenza: An updated cochrane systematic review and meta-analysis. Crit Care Med 2020; 48: e98-e106

[69] Rostaing L, Malvezzi P. Steroid-based therapy and risk of infectious complications. PLoS Med 2016; 13: e1002025

[70] Fardet L, Petersen I, Nazareth I. Common infections in patients prescribed systemic glucocorticoids in primary care: a population-based cohort study. PLoS Med 2016; 13: e1002024

[71] Normand ST. The RECOVERY Platform. N Engl J Med. 2020; DOI: 10.1056/NEJMe2025674:

[72] Group RC, Horby P, Lim WS et al. Dexamethasone in Hospitalized Patients with Covid-19 - Preliminary Report. N Engl J Med. 2020; DOI: 10.1056/NEJMoa2021436

[73] Critchley JA, Carey IM, Harris T et al. Glycemic control and risk of infections among people with type 1 or type 2 diabetes in a large primary care cohort study. Diabetes Care 2018; 41: 2127-2135

[74] Rao Kondapally Seshasai S, Kaptoge S, Thompson A et al. Diabetes mellitus, fasting glucose, and risk of cause-specific death. N Engl J Med 2011; 364: 829-841

[75] Yang JK, Feng Y, Yuan MY et al. Plasma glucose levels and diabetes are independent predictors for mortality and morbidity in patients with SARS. Diabet Med 2006; 23: 623-628

[76] Alqahtani FY, Aleanizy FS, Ali El Hadi Mohamed R et al. Prevalence of comorbidities in cases of Middle East respiratory syndrome coronavirus: a retrospective study. Epidemiol Infect 2018; 147: 1-5

[77] Zhou F, Yu T, Du R et al. Clinical course and risk factors for mortality of adult inpatients with COVID-19 in Wuhan, China: A retrospective cohort study. Lancet 2020; 395: 1054-1062

[78] Barron E, Bakhai C, Kar P et al. Associations of type 1 and type 2 diabetes with COVID-19-related mortality in England: A whole-population study. Lancet Diabetes Endocrinol 2020; 8: 813-822

[79] Holman N, Knighton P, Kar P et al. Risk factors for COVID-19-related mortality in people with type 1 and type 2 diabetes in England: A population-based cohort study. Lancet Diabetes Endocrinol 2020; 8: 823-833
[80] Zhu L, She ZG, Cheng X et al. Association of blood glucose control and outcomes in patients with COVID-19 and pre-existing type 2 diabetes. Cell Metab 2020; 31: 1068-1077 e1063

[81] Kalligeros M, Shehadeh F, Mylona EK et al. Association of obesity with disease severity among patients with coronavirus disease 2019 Obesity (Silver Spring) 2020; 28: 1200-1204

[82] Hamer M, Gale CR, Kivimaki M et al. Overweight, obesity, and risk of hospitalization for COVID-19: A community-based cohort study of adults in the United Kingdom. Proc Natl Acad Sci USA 2020; 117: 21011-21013

[83] Bornstein SR, Rubino F, Khunti K et al. Practical recommendations for the management of diabetes in patients with COVID-19. Lancet Diabetes Endocrinol 2020; 8: 546-550

[84] Erener S. Diabetes, infection risk and COVID-19. Mol Metab 2020; 39: 101044

[85] Feldman-Billard S, Lissak B, Kassaei R et al. Short-term tolerance of pulse methylprednisolone therapy in patients with diabetes mellitus. Ophthalmology 2005; 112: 511-515

[86] Reynolds RM, Labad J, Sears AV et al. Glucocorticoid treatment and impaired mood, memory and metabolism in people with diabetes: The Edinburgh Type 2 Diabetes Study. Eur J Endocrinol 2012; 166: 861-868

[87] Fardet L, Flahault A, Kettaneh A et al. Corticosteroid-induced clinical adverse events: frequency, risk factors and patient's opinion. $\mathrm{Br}$ J Dermatol 2007; 157: 142-148

[88] Sato A, Funder JW, Okubo M et al. Glucocorticoid-induced hypertension in the elderly. Relation to serum calcium and family history of essential hypertension. Am J Hypertens 1995; 8: 823-828

[89] Curtis JR, Westfall AO, Allison et al. Population-based assessment of adverse events associated with long-term glucocorticoid use. Arthritis Rheum 2006; 55: 420-426

[90] Wei L, MacDonald TM, Walker BR. Taking glucocorticoids by prescription is associated with subsequent cardiovascular disease. Ann Intern Med 2004; 141: 764-770

[91] Souverein PC, Berard A, Van Staa TP et al. Use of oral glucocorticoids and risk of cardiovascular and cerebrovascular disease in a population based case-control study. Heart 2004; 90: 859-865 